\title{
INFLUÊNCIA DO EXTRATO HIDROETANÓLICO DAS FOLHAS DE Tropaeolum majus NA RESTAURAÇÃO TECIDUAL EM LESÕES CUTÂNEAS
}

\section{Jéssica Santos Correa \\ Edylaine Aparecida Monteiro \\ Mariana Felgueira Pavanelli \\ Ana Carla Broetto Biazon}

Faculdade Integrado de Campo Mourão, PR, Brasil.

E-mail: anacarlabiazon@gmail.com
RESUMO: O presente estudo teve como objetivo avaliar a ação cicatrizante do extrato hidroetanólico de folhas de Tropaeolum majus em lesões cutâneas em ratos. Para isso foram utilizados 15 ratos Wistar distribuídos em 3 grupos denominados de G7, G14 e G21, de acordo com o período de observação em 7, 14 e 21 dias após o procedimento cirúrgico. Na região dorsal de cada animal foram realizadas duas feridas de $1 \mathrm{~cm}$ de diâmetro, sendo a da esquerda tratada com gel base de Carbopol, e a da direita com gel de Tropaeolum majus. Os tratamentos nas lesões ocorreram diariamente nos períodos citados. Realizou-se estudo comparativo entre os dois grupos por meio da análise micro e macroscópica. Observou-se formação de crosta em todas as feridas até o sétimo dia. Na avaliação histológica, as lesões tratadas com o extrato de Tropaeolum majus apresentaram uma menor quantidade de infiltrado inflamatório e de proliferação fibloblástica com relação às lesões tratadas com o gel base e uma maior colageneização bem como mais acentuada angiogênese no $7^{\circ}, 14^{\circ}$ e $21^{\circ}$ dia do pós-operatório. Conclui-se que o extrato hidroetanólico de folhas de Tropaeolum majus promoveu melhora do processo de cicatrização por aumento da formação de neovasos e colageneização.

PALAVRAS-CHAVE: Cicatrização; Plantas Medicinais; Tropaeolum majus.

\section{INFLUENCE OF HYDROETHANOLIC EXTRACT FROM THE LEAVES OF Tropaeolum majus FOR TISSUE RESTORATION OF SKIN LESIONS}

\begin{abstract}
The healing activity of the hydroethanolic extract of Tropaeolum majus leaves on rats' skin lesions is provided. Fifteen Wistar rats were used, distributed in three groups, G7, G14 and G21, according to observation period 7, 14 and 21 days after surgery. Two wounds, diameter $1 \mathrm{~cm}$, on the dorsal region of each animal, were treated with Carbopol gel on the left side and by Tropaeolum majus on the right side. Lesions were treated daily during the periods above. A comparative study was undertaken between the two groups by microand macroscopy. The formation of a wound scab on all wounds up to the seventh day was observed. Histological assessment revealed that lesions treated with Tropaeolum majus extract had a lower inflammatory infiltrate rate and fibroblastic proliferation when compared to lesions treated with gel, coupled to a greater collagenization and deeper angiogenesis on the $7^{\text {th }}, 14^{\text {th }}$ and $21^{\text {st }}$ day after surgery. Results showed that hydroethanolic extract of Tropaeolum majus leaves caused a better improvement in the healing process through the increase in the formation of neo-vases and collagenization.
\end{abstract}

KEY WORDS: Wound Healing; Medicinal Plants; Tropaeolum majus. 


\section{INTRODUÇão}

As plantas medicinais, desde a antiguidade, têm sido muito utilizadas para o tratamento de diversas doenças, bem como para a pesquisa e desenvolvimento de novos farmácos. Sabe-se que mais de 20.000 espécies são utilizadas na medicina tradicional, no entanto muitos efeitos terapêuticos não são comprovados cientificamente (GUPTA et al., 2008).

Embora a indústria farmacêutica tenha obtido avanços consideráveis no que diz respeito à disponibilidade de drogas capazes de estimular o processo de cicatrização, apenas 1 a 3\% de todos os medicamentos listados em farmacopéias ocidentais são destinados para o uso na pele ou feridas. Destes, pelo menos $1 / 3$ são obtidos de plantas medicinais (CSUPOR et al., 2010).

As feridas resultam de uma agressão física à pele que causa uma abertura ou ruptura da mesma originando uma lesão tecidual (SCHMIDT et al., 2009). Logo após a lesão tem início o processo de cicatrização, um processo fisiológico que envolve uma complexa sequência de interações entre vários tipos celulares e citocinas com o objetivo de devolver ao tecido danificado sua função e integridade (SCHMIDT et al., 2009; PATHER et al., 2010).

A cicatrização de feridas inicia-se com uma resposta inflamatória que tem como características o aumento do fluxo sanguíneo e da permeabilidade capilar e a migração de leucócitos para a região lesada (EURIDES et al., 1996). Esta é a primeira fase do processo de cicatrização que vem seguida pela fase de proliferação de fibroblastos e deposição da matriz extracelular e, por fim, ocorre a fase de remodelação tecidual (PESSOA et al., 2012).

O tratamento de feridas tem como objetivo principal que a cura da lesão seja obtida no menor tempo possível com o mínimo de dor e desconforto para o paciente e que no local da ferida seja obtida uma cicatriz fina e flexível, com máxima resistência à tração (PATHER et al., 2010).

Diversos estudos biológicos já foram conduzidos para avaliar ação cicatrizante em diferentes espécies vegetais (FALEIRO et al., 2014; MACHADO et al., 2011; RATH et al., 2006). Plantas com atividade anti-inflamatória podem ter ação direta na cicatrização de feridas, da mesma forma como extratos de plantas com ação antimicrobiana podem evitar a contaminaçao das lesões (CSUPOR et al., 2010).

Tropaeolum majus L. (Tropaeolaceae) é uma importante planta medicinal nativa da América do Sul (GOMES et al., 2012). A espécie é amplamente distribuída por todo o mundo e no Brasil é conhecida popularmente como chaguinha, capuchina e nastúrcio (LOURENÇO, 2012a). Na medicina popular, as folhas de Tropaeolum majus são utilizadas para o tratamento de diversas doenças como distúrbios cardiovasculares, infecção do trato urinário, asma e constipação (GOMES et al., 2012).

A planta também é considerada anti-inflamatória, antisséptica, anti-escorbútica e antimicrobiana (ZANETTI, 2003; LOURENÇO, 2011). Estudosfitoquímicosapontaram a presença de ácidos graxos (ácido erúcico, ácido oléico e linoléico), benzil isotiocianato, glucosinolatos e flavonóides (isoquercitrina, quercetina e campferol) nas folhas de Tropaeolum majus (LOURENÇO, 2012b).

$\mathrm{Na}$ literatura consultada até o momento, não foi encontrado nenhum estudo sobre a ação do extrato de Tropaeolum majus na reparação tecidual em lesões cutâneas. Assim, considerando os efeitos farmacológicos da planta e a necessidade da descoberta de novas substâncias com potencial cicatrizante, o presente trabalho objetivou avaliar o efeito da utilização do extrato hidroetanólico das folhas de Tropaeolum majus na reparação tecidual de ratos Wistar.

\section{METODOLOGIA}

\subsection{DELINEAMENTO EXPERIMENTAL}

O protocolo experimental deste estudo seguiu os princípios éticos na experimentação animal adotados pelo Conselho Nacional de Controle de Experimentação Animal (CONCEA) e foi aprovado pela Comissão de Ética em Experimentação Animal da Faculdade Integrado de Campo Mourão (CEEA), sob o protocolo de número 779.

Foram utilizados 15 ratos (Rattus Norvegicus albinus) da linhagem Wistar, machos, procedentes do biotério central da Universidade Estadual de Maringá. 
Os ratos foram aclimatados por um período de sete dias no Biotério da Faculdade Integrado de Campo Mourão e divididos em três grupos de 5 animais cada, conforme o período de experimentação. Os grupos foram denominados como G7, G14 e G21, de acordo com o período de avaliação em 7, 14 e 21 dias do pós-operatório, respectivamente. Os animais permaneceram isolados individualmente em gaiolas plásticas padrão. Eles foram mantidos em temperatura e umidade controlada em ciclo circadiano (claro/escuro de $12 \mathrm{em} 12$ horas). Os animais receberam $60 \mathrm{~g}$ de ração/dia com livre acesso à água.

\subsection{PREPARO DO EXTRATO}

As folhas de Tropaeolum majus foram adquiridas na cidade de Campo Limpo Paulista (SP) no início de março de 2014. A planta foi catalogada em exsicata sob o número 1080 no Herbário da Faculdade Integrado de Campo Mourão (PR).

As folhas foram secas naturalmente, à temperatura ambiente, sob papel craft. Posteriormente, as folhas foram colocadas em estufa de secagem na temperatura de $36{ }^{\circ} \mathrm{C}$ durante $24 \mathrm{~h}$ para retirada total da umidade.

O extrato hidroetanólico das folhas de Tropaeolum majus $L$. foi preparado utilizando $700 \mathrm{~g}$ de folhas desidratadas e trituradas em processador doméstico. Após foi preparado uma solução 10\% em álcool 70\% e colocado em shaker por 5 horas com 130 rotações por minuto. Após, o extrato hidroalcoólico foi filtrado a vácuo e passado em rota evaporador para remoção do etanol sendo, em seguida, liofilizado para estocagem a $-20^{\circ} \mathrm{C}$.

\subsection{PREPARO DOS GÉIS}

O gel base utilizado para a incorporação do extrato foi o gel de Carbopol 940 a $2 \%$. O gel foi preparado utilizando $98 \mathrm{ml}$ de água a $60^{\circ} \mathrm{C}$ e $2 \mathrm{~g}$ de Carbopol 940. A mistura foi homogeneizada até que a mesma chegasse à consistência de gel. Após, $7 \mathrm{~g}$ do extrato liofilizado de Tropaeolum majus foi incorporado a $70 \mathrm{~g}$ do gel base obtendo-se, assim, um gel do extrato de Tropaeolum majus a $10 \%$. Os géis foram mantidos em refrigeração até o uso.

\subsection{PROCEDIMENTO CIRÚRGICO}

Após a anestesia geral, cada rato foi posicionado em decúbito ventral, imobilizado em prancha operatória e submetido a epilação na região dorsal em área de 6 $\mathrm{cm}^{2}$. Para a demarcação da pele a ser retirada, foi utilizado um punch metálico com lâmina cortante na sua borda inferior. Em seguida foram retirados dois fragmentos cutâneos (lesões) com $1 \mathrm{~cm}$ de diâmetro cada no centro da área epilada, até a exposição da fáscia muscular dorsal. A hemostasia foi feita por compressão digital com gaze.

Em todos os grupos, a lesão da esquerda foi padronizada como sendo controle e a lesão da direita, extrato de Tropaeolum majus. Todas as lesões foram tratadas diariamente e no mesmo horário nos respectivos grupos. As lesões controle receberam $0,5 \mathrm{~g}$ de gel base (Carbopol) e as demais lesões receberam 0,5 $\mathrm{g}$ de gel de Carbopol com $10 \%$ de extrato hidroetanólico de folhas de Tropaeolum majus.

\subsection{AVALIAÇÃO MACROSCÓPICA}

A avaliação macroscópica foi realizada por meio da medição dos diâmetros inicial e final das lesões de cada animal com paquímetro digital graduado em milímetros (Figura 1). Os dados foram anotados em ficha protocolo para posterior comparação. As medidas foram realizadas em todos os grupos de animais.

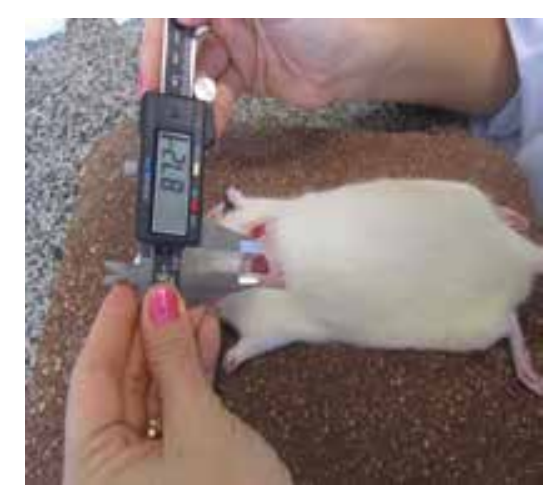

Figura 1. Avaliação macroscópica do diâmetro da lesão com paquímetro digital

\subsection{PROCESSAMENTO HISTOLÓGICO}

Após o período de tratamento das lesões, os animais foram eutanasiados com injeção de $\mathrm{KCl}$ 
intracardíaco após a anestesia dissociativa do animal. A peça cirúrgica foi retirada logo após a morte do animal, sendo constituída da cicatriz ou lesão cutânea, com margem de $1 \mathrm{~cm}$ de pele em torno da lesão, com profundidade até a musculatura dorsal do animal. A partir disso, o material foi fixado em formol a $10 \%$ por 24 horas, após realizou-se no Laboratório de Patologia Animal da Faculdade Integrado de Campo Mourão a técnica histológica de rotina incluindo as etapas de desidratação gradativas, diafanização, infiltração e emblocamento em parafina das amostras. A partir de cada bloco de parafina foram obtidos cortes histológicos da lesão de cada animal posteriormente coradas pela técnica de HematoxilinaEosina.

\subsection{AVALIAÇÃO MICROSCÓPICA}

As análises dos cortes histológicos foram realizadas em microscópio óptico tetraocular. Toda a extensão do fragmento da ferida cutânea foi avaliada. Os critérios histológicos avaliados incluíram infiltrados inflamatórios, proliferação fibroblástica, colageneização e angiogênese os quais foram avaliados em escores, definidos de acordo com focos/corte. Os resultados destes escores foram transcritos em cruzes onde cada critério teve uma forma de avaliação conforme o quadro a seguir:

Quadro 1. Escores empregados na avaliação dos critérios infiltrado inflamatório, proliferação fibroblástica e angiogênese

\begin{tabular}{|c|c|c|c|}
\hline Escores & Infiltrado inflamatório & Proliferação fibroblástica & Angiogênese \\
\hline+ & Até 2 focos/corte & Até 10 células/campo & Até 10 células/campo \\
\hline++ & 3 a 5 focos/corte & 11 a 30 células/campo & 11 a 30 células/campo \\
\hline+++ & $>$ que 6 focos/corte & $>$ que 31 células/campo & $>$ que 31 células/campo \\
\hline
\end{tabular}

No critério de colageneização não há como quantificar estes escores, devido à dificuldade de delimitar a fibra colágena, o que tornou este critério subjetivo.

\subsection{ANÁLISE ESTATÍSTICA}

Os dados foram analisados utilizando-se o programa GraphPadPrism 5 e foi aplicado teste $t$ de Student.

\section{RESULTADOS E DISCUSSÃO}

O uso de substâncias tópicas para auxiliar no processo de reparação tecidual tem sido muito estudado. A utilização de plantas para a manutenção da saúde vem recebendo muitos incentivos econômicos, sociais e até mesmo da Organização Mundial da Saúde (OMS) (SANTOS, 2006).

No presente estudo, avaliou-se a influência do extrato hidroetanólico das folhas de Tropaeolum majus na cicatrização de lesões cutâneas em ratos tanto no aspecto macroscópico quanto no aspecto microscópico. Conforme realizado no trabalho de Amorim (2006), a ferida foi induzida no dorso do animal para que não houvesse interferência no processo de cicatrização. A lesão tecidual foi avaliada no $7^{\circ}, 14^{\circ}$ e $21^{\circ}$ dias após a cirurgia, pois estes são considerados os dias mais significantes para estudo do mecanismo de cicatrização na pele de ratos (MIRANDA, 2001).

A forma farmacêutica utilizada foi a de gel, definida como uma preparação obtida da dispersão de polímeros gelificantes em meio aquoso (VILLANOVA, 2010). Os géis são utilizados para incorporação de substâncias a serem aplicadas em cicatrizes, pois podem ser utilizados em várias fases da cicatrização, devido ao fato de não danificar o tecido de granulação, favorecer a angiogênese, promover a quimiotaxia dos leucócitos e manter o meio úmido (MANDELBAUM, 2003).

$\mathrm{Na}$ avaliação macroscópica, observou-se que nenhum animal apresentou secreção purulenta na ferida e houve formação de crosta, que é resultado do preenchimento da lesão por coágulos de fibrina e exsudatos (SANTOS et al., 2006) em todos os animais do 
grupo G7. Comparando as lesões controle e extrato de Tropaeolum majus, nota-se que houve diferenças entre elas, já que as lesões tratadas com o extrato de Tropaeolum majus apresentaram crosta mais espessa, indicando assim que ocorreu uma reparação tecidual mais acelerada.

No grupo G14, os animais também apresentaram ferida sem secreção purulenta. Houve uma evolução na reparação tecidual em ambas as lesões, porém, nenhuma das lesões havia cicatrizado. No grupo G21, a restauração tecidual apresentou-se quase completa sendo que uma das lesões controle já estava fechada, assim como duas lesões extrato de Tropaeolum majus. Resultados diferentes foram encontrados em um estudo anterior (SANTOS et al., 2006) onde as lesões se encontravam totalmente fechadas em todos os animais no $21^{\circ}$ dia do pós-operatório.
A medida do diâmetro da lesão realizada por paquímetro digital mostra que houve uma diminuição significativa no tamanho da lesão extrato do grupo G7 para o mesmo grupo de lesões controle $(+69 \%)$. Nos demais grupos estudados, apesar da evolução do processo de cicatrização, não foi encontrada diferença estatisticamente significativa entre as lesões do controle negativo e extrato de Tropaeolum majus (Tabela 1). Resultados semelhantes a este foram encontrados em um outro estudo realizado (AMORIM et al., 2006), no qual houve diferença significativa nas médias de 7 dias verificadas em favor do grupo experimento com extrato aquoso de Orbignya phalerata comparado com o grupo controle.

Tabela 1. Paquimetria da diferença das medidas inicial e final do diâmetro das lesões controle (CONT) e extrato de Tropaeolum majus (EXT) nos grupos G7, G14 e G21

\begin{tabular}{l|c|c|c|c|c|c}
\hline \multirow{2}{*}{ ANIMAL } & \multicolumn{2}{|c|}{7 dias } & \multicolumn{2}{c}{14 dias } & \multicolumn{2}{c}{21 dias } \\
\cline { 2 - 7 } & CONT. & EXT. & CONT. & EXT. & CONT. & EXT. \\
\hline RATO 1 & 0,79 & 2,87 & 6,34 & 6,74 & 5,37 & 8,32 \\
RATO 2 & 2,35 & 2,88 & 5,95 & 7,76 & 7,68 & 9,01 \\
RATO 3 & 2,62 & 2,77 & 6,63 & 7,66 & 8,07 & 9,51 \\
RATO 4 & 1,37 & 2,42 & 5,93 & 6,11 & 6,90 & 9,32 \\
RATO 5 & 1,17 & 3,03 & 6,30 & 5,67 & 10,13 & 5,40 \\
\hline MÉDIA \pm EPM & $1,65 \pm 0,31$ & $2,79 \pm 0,09 *$ & $6,23 \pm 0,11$ & $6,78 \pm 0,36$ & $7,63 \pm 0,69$ & $8,31 \pm 0,67$ \\
\hline
\end{tabular}

$* \mathrm{p}<0,05$.

O processo de cicatrização tecidual, indispensável para a sobrevivência dos seres vivos é composto por várias fases (BALBINO et al., 2005). A coagulação, primeira fase do processo de cicatrização, tem início imediatamente após a lesão e tem como função evitar a perda excessiva de sangue. Essa fase depende da atividade plaquetária e da cascata de coagulação. Substâncias vasoativas, proteínas adesivas, fatores de crescimento e proteases são liberadas e participam do desencadeamento de outras fases (MANDELBAUM et al., 2003). Nenhum animal apresentou hemorragia durante os tratamentos sendo que a hemostasia no pós-operatório, realizada por compressão digital com gaze, foi o suficiente para parar o sangramento.
$\mathrm{Na}$ segunda fase, conhecida como fase inflamatória, ocorre primeiramente a migração de neutrófilos dos vasos sanguíneos para a ferida mediada por glicoproteínas como a selectina. Após, os monócitos se infiltram em respostas a estímulos parecidos aos dos neutrófilos e se diferenciam em macrófagos. Estes dois tipos celulares (neutrófilos e macrófagos) são os responsáveis por remover partículas estranhas, bactérias e outros tecidos mortos da ferida. Os macrófagos também são importantes por produzirem uma diversidade de fatores de crescimento como os EGF e FGF (fatores de crescimento dos fibroblastos) (ISSAC et al., 2010).

$\mathrm{Na}$ análise microscópica, com relação à presença de infiltrados inflamatórios, observou-se no grupo 
G7, em ambas as lesões, uma discreta presença destes achados. Nos demais grupos, a presença de infiltrados inflamatórios foi praticamente insignificante. Como comprovado em outro estudo (LOURENÇO, 2011), o extrato de Tropaeolum majus tem ação anti-inflamatória o que pode explicar o número pequeno de infiltrados inflamatórios nas lesões tratadas com extrato de Tropaeolum majus.

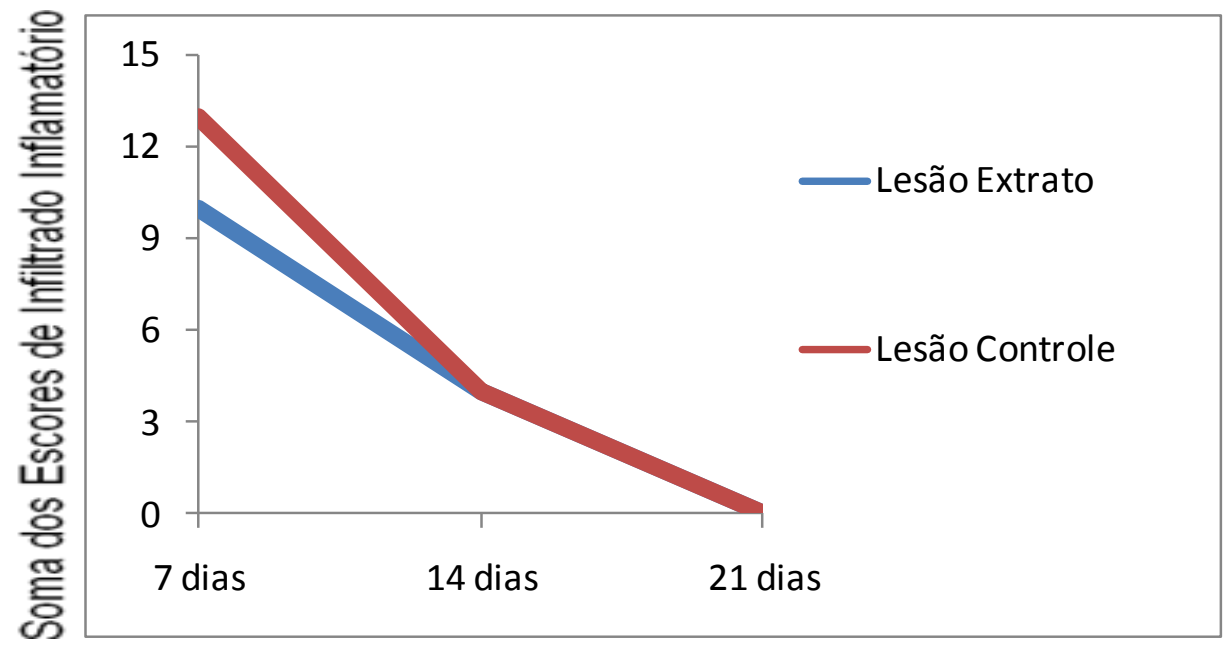

Figura 2. Presença de infiltrados inflamatórios na cicatrização de feridas cutâneas tratadas com extrato de Tropaeolum majus (EXT) e gel base (controle - CONT), para os grupos G7, G14, G21.

*A soma dos escores de infiltrados inflamatórios foi realizada mediante critério descrito na metodologia.

A fase fibroblástica, terceira fase do processo de cicatrização, é caracterizada pelo aumento do número de fibroblastos que são as principais células envolvidas na cicatrização e têm como função manter a integridade do tecido conjuntivo. Os fibroblastos são estimulados a produzir e a depositar componentes da matriz extracelular, acontecimento necessário para permitir e promover a reepitelização do tecido (PAGNANO et al., 2008). Os fibroblastos são os principais responsáveis pela produção de colágeno no local da lesão. Para que haja a eficiência da fibroplasia é necessário que ocorra em paralelo a formação de novos vasos sanguíneos (BALBINO et al., 2005).

Quanto à presença de fibroblastos, observou-se a mesma evolução em ambas as lesões no grupo G7. Nos grupos G14 e G21, as lesões controle apresentaram maior proliferação fibroblástica (Tabela 2) em relação às outras lesões.

Tabela 2. Escores da presença de fibroblastos na cicatrização de feridas cutâneas, tratadas com extrato de Tropaeolum majus (EXT) e gel base (controle negativo - CONT), para os grupos G7, G14 e G21

\begin{tabular}{l|c|c|c|c|c|c}
\hline \multirow{2}{*}{ ESCORES } & \multicolumn{2}{|c|}{7 dias } & \multicolumn{2}{c}{14 dias } & \multicolumn{2}{c}{21 dias } \\
\cline { 2 - 7 } & CONT. & EXT. & CONT. & EXT. & CONT. & EXT. \\
\hline $0(0=$ ausente $)$ & 0 & 0 & 0 & 0 & 0 \\
$+(1=$ discreto $)$ & 2 & 2 & 0 & 2 & 3 & 4 \\
$++(2=$ moderado $)$ & 3 & 3 & 5 & 0 & 0 & 0 \\
$++(3=$ acentuado $)$ & 0 & 0 & 5 & 5 & 5 & 5 \\
\hline Total & 5 & 5 & 5 & 5 & 5 \\
\hline
\end{tabular}


A angiogênese, quarta fase do processo de cicatrização, é essencial para o suprimento de oxigênio e nutrientes para o novo tecido que está se formando (MANDEBAUM et al., 2003). Com relação à angiogênese, o presente estudo mostrou que a formação de neovasos nas lesões extrato de Tropaeolum majus no grupo G7 variou entre moderado e acentuado sendo que o mesmo aconteceu com o grupo G14; já no grupo G21 estes achados variaram de discreto a moderado. Nas lesões controle, no grupo G7, os achados variaram de discreto a ausente, no grupo G14 de discreto a acentuado e no grupo G21 estes achados foram considerados discretos (Tabela 3). Estes resultados mostram uma maior angiogênese nas lesões tratadas com Tropaeolum majus.

Tabela 3. Escores da presença de neovasos na cicatrização de feridas cutâneas, tratadas com extrato de Tropaeolum majus (EXT) e gel base (controle negativo - CONT), para os grupos G7, G14 e G21

\begin{tabular}{|c|c|c|c|c|c|c|}
\hline \multirow{2}{*}{ Escores } & \multicolumn{2}{|c|}{7 dias } & \multicolumn{2}{|c|}{14 dias } & \multicolumn{2}{|c|}{21 dias } \\
\hline & CONT. & EXT. & CONT. & EXT. & CONT. & EXT. \\
\hline $0(0=$ ausente $)$ & 2 & 1 & 0 & 0 & 0 & 0 \\
\hline$+(1=$ discreto $)$ & 3 & 0 & 3 & 1 & 5 & 2 \\
\hline$++(2=$ moderado $)$ & 0 & 2 & 0 & 2 & 0 & 3 \\
\hline$+++(3=$ acentuado $)$ & 0 & 2 & 2 & 2 & 0 & 0 \\
\hline Total & 5 & 5 & 5 & 5 & 5 & 5 \\
\hline
\end{tabular}

A última fase do processo de cicatrização é a colageneização, fase em que ocorre a maturação e remodelagem da matriz extracelular. Esta etapa é caracterizada pela grande e acelerada deposição de colágeno na lesão e é durante este período que a cicatriz adquire sua máxima resistência tênsil (BALBINOA et al., 2005).

Com relação à colageneização, observou-se uma colageneização acentuada $(+++)$ nas lesões do extrato de Tropaeolum majus em todos os grupos (G7, G14 e
G21); nas lesões controle, estes achados variaram de discreto a moderado nos grupos avaliados (Tabela 4). Resultados semelhantes foram encontrados em outro estudo realizado (SANTOS et al., 2006), no qual a análise histológica demonstrou que a colageneização obteve resultado significativo no $7^{\circ}$ e $14^{\circ}$ dias do pós-operatório, com intensificação do processo na lesões tratadas com extrato de Jatropha gossypiifolia l. comparado ao grupo controle.

Tabela 4. Escores da presença de colágeno na cicatrização de feridas cutâneas, segundo a utilização do extrato de Tropaeolum majus (extrato - EXT) e gel base (controle negativo - CONT), durante o período de sete (grupo G7), quatorze (grupo G14) e vinte e um dias (grupo G21)

\begin{tabular}{l|c|c|c|c|c|c}
\hline \multirow{2}{*}{ Escores } & \multicolumn{2}{|c|}{7 dias } & \multicolumn{2}{c|}{14 dias } & \multicolumn{2}{c}{21 dias } \\
\cline { 2 - 7 } & CONT. & EXT. & CONT. & EXT. & CONT. & EXT. \\
\hline 0 (0=ausente) & 0 & 0 & 0 & 0 & 0 & 0 \\
$+(1=$ discreto $)$ & 4 & 0 & 0 & 1 & 0 & 0 \\
$++(2=$ moderado $)$ & 1 & 1 & 5 & 2 & 1 & 0 \\
$+++(3=$ acentuado $)$ & 0 & 4 & 0 & 2 & 4 & 5 \\
\hline Total & 5 & 5 & 5 & 5 & 5 & 5 \\
\hline
\end{tabular}


Desta forma, a colageneização e a angiogênese foram mais evidentes nas lesões tratadas com Tropaeolum majus em relação às lesões controle, o que sugere que o processo de cicatrização na presença de extrato hidroetanólico de Tropaeolum majus foi facilitado pela utilização do referido extrato.

\section{CONCLUSÃO}

Os resultados obtidos no presente estudo mostraram que a utilização do extrato hidroetanólico das folhas de Tropaeolum majus auxiliou no processo de cicatrização tanto no aspecto macroscópico quanto no aspecto microscópico de avaliação. Novos estudos podem ser realizados utilizando técnicas diferentes de coloração para comprovar a eficácia da ação cicatrizante e investigar os mecanismos presentes neste efeito para o desenvolvimento de um novo fitoterápico.

\section{REFERÊNCIAS}

AMORIM, E.; MATIAS, J. E. F.; COELHO, J. C. U.; CAMPOS, A. C. L.; STAHLKE JR, H. J.; TIMI, J. R. R.; ROCHA, L. C. A.; MOREIRA, A. T. R.; RISPOLI, D. Z.; FERREIRA, L. M. Efeito do uso tópico do extrato aquoso de Orbignyaphalerata (Babaçu) na cicatrização de feridas cutâneas - estudo controlado em ratos. Acta Cir. Bras., v. 21, n. 6, p. 66-76, 2006.

BALBINO, C. A.; PEREIRA, L. M.; CURI, R. Mecanismos envolvidos na cicatrização: uma revisão. Rev. Bras. Cienc. Farm., v. 41, n. 1, 2005.

CSUPOR, D.; BLAZSÓ, G.; BALOGH, A.; HOHMANN, J. The traditional Hungarian medicinal plant Centaurea SadlerianaJanka accelerates wound healing in rats. $\mathbf{J}$ Ethnopharmacol, v. 127, p. 193-195, 2010.

EURIDES, D.; MAZZANTI, A.; BELLETI, M. E.; SILVA, L. A. F. DA; FIORAVANTE, M. C. S.; TRONCOSO NETO, N. S.; CAMPOS, V. A. DE; LEMOS, R. C.; SILVESTRINI JUNIOR, P. L. Morfologia e morfometria da reparação tecidual de feridas cutâneas de camundongos tratadas com solução aquosa de barbatimão (stryphynodendron barbatiman martius). Rev FZVA, Uruguaiana, v. 3, n. 1, p. 30-40, 1996.

FALEIRO, C. C.; ELIAS, S. T.H.; CAVALCANTI, L. C. CAVALCANTI, Á. S. S. O extrato das folhas de babosa, Aloe vera na cicatrização de feridas experimentais em pele de ratos, num ensaio controlado por placebo. Natureza on line. Disponível em: <http://www.naturezaonline.com. br>. Acesso em: 25 mar. 2014.

GOMES, C.; LOURENÇO, E. L.; LIUTI, É. B.; DUQUE, A. O.; NIHI, F.; LOURENÇO, A. C.; MENDES, T. C.; GASPAROTTO JUNIOR, A.; DALSENTER, P. R. Evaluation of subchronic toxicity of the hydroethanolic extract of Tropaeolum majus in Wistar rats. J Ethnopharmacol, v. 142, p. 481-487, 2012.

GUPTA, D.; BLEAKLEY, B.; GUPTA, R. K. Dragon's blood: botany chemistry and therapeutic uses. J Ethnopharmacol, v. 115, p. 361-380, 2008.

ISAAC, C.; LADEIRA, P. R. S. de; RÊGO, F. M. P. do; ALDUNATE, J. C. B.; FERREIRA, M. C. Processo de cura das feridas: cicatrização fisiológica. Rev. Medicina (USP), São Paulo, v. 89, p. 125-131, 2010.

LOURENÇO, E. L. Atividade de Tropaeolum majus $l$. sobre a mobilização e migração leucocitária em modelo de bolsão inflamatório. Arq Ciênc Saúde UNIPAR, Umuarama, v. 15, n. 3, p. 243-256, set./dez. 2011.

LOURENÇO, E. L. Toxicologia reprodutiva do extrato hidroalcoolico liofilizado da Tropaeolum majus L. (chaguinha). 2012. 108f. Tese (Doutorado em farmacologia) - Universidade Tecnológica Federal do Paraná, Curitiba, 2012a.

LOURENÇO, E. L.; MULLER,J. C.; BOARETO,A. C.; GOMES, C.; LOURENÇO, A. C.; MINATOVICZ, B.; CRESTANI, S.; GASPAROTTO, A. J.R.; MARTINO-ANDRADE, A. J.; DALSENTER, P. R. Screening for in vivo (anti) estrogenic and (anti) androgenic activities of Tropaeolum majus $L$. and its effect on uterine contractility. J Ethnopharmacol., v. 141, p. 418-423, 2012b.

MACHADO, A.C.; DEZAN JUNIOR, E.; GOMES-FILHO, J. E.; CINTRA, L. T. A.; RUVIÉRE, D. B.; ZOCCAL, R.; DAMANTE, C., A.; JARDIM JUNIOR, E. G. Evaluation of 
tissue reaction to Aroeira (Myracrodruonurundeuva) extracts: a histologicand edemogenic study. J. Appl. Oral Sci., v. 20, p. 414-418, 2011.

MANDELBAUM, S. H; DI SANTIS, É. P.; MANDELBAUM, M. H. S. Cicatrização: conceitos atuais e recursos auxiliares Parte I. An bras Dermatol., Rio de Janeiro, v. 78, n. 4, p. 393-410, jul./ago. 2003.

MIRANDA, L. T. G. S. Uso da tintura de Arnica em feridas cutâneas abertas em ratos. 2001. 38f. Tese (Doutorado) - Universidade Federal de São Paulo, Escola Paulista de Medicina, São Paulo, 2001.

PAGNANO, L. O.; BARALDI-ARTONI, S. M.; PACHECO, M. R.; SANTOS, E. DOS; OLIVEIRA, D.; LUI, J. F. Morfometria de fibroblastos e fibrócitos durante o processo cicatricial na pele de coelhos da raça Nova Zelândia Branco tratados com calêndula. Cienc. Rural, v. 38, n. 6, p. 1662-1666, set. 2008.

PATHER, N.; VILJON, A. M.; KRAMER, B. A biochemical comparison of the in vivo effects of Bulbine frutescens and Bulbine natalensis on cutaneous wound healing. $\mathbf{J}$ Ethnopharmacol, v. 133, p. 364-370, out. 2010.

PESSOA, W. S.; ESTEVÃO, L.R. M.; SIMÕES, R. S.; BARROS, M. E. G. DE; MENDONÇA, F. S.; BARATELLAEVÊNCIO, L.; EVÊNCIO-NETO, J. Effects of angico extract (Anadenantheracolubrina var. cebil) in cutaneous wound healing in rats. Acta Cir. Bras., v. 27, n. 10, p. 665-671, 2012.

RATH, B. S.; BODHANKAR, S. L.; BAHETI, A. M. Evaluation aqueous leaves extract of Moringa oleifera linn for wound healing in albino rats. Indian J Exp Biol., v.44, n. 11, p. 898-901, 2006.

SANTOS, M.F.S.; CZECZKO, N. G.; NASSIF, P. A. N.; RIBAS-FILHO, J. M.; ALENCAR, B. L. F. de; MALAFAIA, O.; RIBAS, C. A. P. M.; TRAUTWEIN, V. M.; HENRIQUES, G. S.; MAIA, J. M. A.; BITTENCOURT, R. C. A. Avaliação do uso do extrato bruto de Jatropha gossypiifolia $l$. na cicatrização de feridas cutâneas em ratos. Acta Cir. Bras., v. 21, suppl. 3, 2006.

SCHMIDT, C.; FRONZA, M.; GOETTERT, M.; GELLER, F.;
LUIK, S.; FLORES, E. M.; BITTENCOURT, C. F.; ZANETTI, G. D.; HEINZMANN, B. M.; LAUFER, S.; MERFORT, I. Biological studies on Brazilian plants used in wound healing. J Ethnopharmacol., v. 122, n. 3, p. 523-532, fev. 2009.

VILLANOVA, J. C. O.; ORÉFICE, R. L.; CUNHA, A. S. Aplicações Farmacêuticas de Polímeros. Polímeros, v. 20, n. 1, p. 51-64, 2010.

ZANETTI, G. D; MANFRON, M. P.; HOELZEL, S. C. S. M.; PAGLIARIN, V. P.; MOREL, A. F. Toxicidade Aguda e Atividade Antibacteriana dos Extratos de Tropaeolum majus L. Acta Farm. Bonaerense, Buenos Aires, v. 22, n. 2, p. 159-162, jan. 2003.

Recebido: 03 março de 2016

Revisado: 06 abril de 2016 Aceito: 14 abril de 2016 